\title{
The Properties of Nanohydroxyapatite Materials and its Biological Effects
}

\author{
Xiaofeng Pang ${ }^{1,2}$, Hongjuan Zeng ${ }^{1}$, Jialie Liu ${ }^{1}$, Shicheng Wei ${ }^{3}$, Yufeng Zheng ${ }^{3}$ \\ ${ }^{1}$ Institute of Life Science and Technology, University of Electronic Science and Technology of China, Chengdu, China; ${ }^{2}$ International \\ Centre for Materials Physics, Chinese Academy of Science, Shenyuning, China; ${ }^{3}$ School of Stomatology, Peking University, Beijing, \\ China. \\ Email: pangxf@mail.sc.cninfo.net
}

Received March $3^{\text {rd }}, 2010$; revised April 30 ${ }^{\text {th }}, 2010$; accepted May $2^{\text {nd }}, 2010$.

\begin{abstract}
The nanohydroxyapatites (HAP) and its biological effects have been studied using ultraviolet absorption spectrum, $X$-ray diffraction $(X R D)$ structure analysis, fluorescent and infrared spectrum of absorption and MTT method. The nanohydroxyapatites are prepared and made by using Sol-gel method, in which the parameters of process and reaction are controlled as: $P H>9, C a / P=1.67$, sintering temperature of $1100^{\circ} \mathrm{C}$ and sintering time 2 hours. The results of the study show that nanohydroxyapatites can absorb the amino acid molecules, the absorption is better for stronger acidity of amino acids. We also find that the nanohydroxyapatites and complex of nanoHAP+ nanoCrO $\mathrm{O}_{2}$ can all restrain the proliferation of cells, but their toxiciteis are all first degree or minor, but the restrained effect of the latter is smaller than that of the former, although they can decrease the relative proliferation rate of cells. The nanohydroxyapatites can also change the molecular structure of human serum albumin.
\end{abstract}

Keywords: Nanohydroxyapatite, Cell, Biological Effect, Toxicity, Amino Acids, Sol-Gel

\section{Introduction}

As it is known, the ceramic materials and metallic materials, such as $\mathrm{Ni}-\mathrm{Cr}$ and $\mathrm{Co}-\mathrm{Cr}$ alloyes serve often as a fixed dental materials of restoration in human bones, teeth and vascular stents, but they have a lot of shortcomings, such as there are $\mathrm{Ni}$ and $\mathrm{Cr}$ hypersusceptibility for some men and ion exchange between the material and tooth, which lead the distortion and discoloration of the gingival edge and affect also its appearance. Hydroxyapatite (HAP) is now the main inorganic components in human bones and teeth, and has excellent biocompatibility, biological activity and bone conduction. As matter of fact, the bone is a kind of flexible and toughening polymer composed of hydroxyapatite-based composites. The composition and structure of artificial-hydroxyapatite are similar to inorganic components in human bone tissues, it has a nontoxicity, non-irritant property, and is also non-allergenic, non-mutagenic and carcinogenic, and can generate a chemical reaction with bone to change bone conduction. Therefore, nano-hydroxyapatites could be widely used in biological hard tissues, such as human bones, teeth and vascular stents, as repair and replacement materials, such as in oral implantology for increasing dental spine, ear bone or spine replacement, etc., allowing better results compared to those using metals and polymers [1-6]. However, the biological effects of nano-hydroxyapatites and its toxicity have been not studied systematically as yet. Therefore, it is very necessary to investigate their biological effects and toxicity. This paper will describe a study of this problem by a novel method using light spectrum technique.

Crystalline hydroxyapatite belongs to hexagonal system, has a $\mathrm{L}_{6} \mathrm{PC}$ symmetry and $P_{6}^{3} / \mathrm{m}$ space group, its structure is hexagonal prism, with the surface of hexagon which is perpendicular to c-axis, angle between a and b-axes is $120^{\circ}$, parameters of its crystalline cell are $\mathrm{a}_{0}=$ $\mathrm{b}_{0}=0.943 \sim 0.938 \mathrm{~nm}, \mathrm{c}_{0}=0.688 \sim 0.686 \mathrm{~nm}$, the unit cell contains $10 \mathrm{Ca}^{2+}, 6 \mathrm{PO}_{4}^{3-}$ and $2 \mathrm{OH}^{-} . \mathrm{OH}^{-}$in the cell is in the four corners, $10 \mathrm{Ca}^{2+}$ occupy two kinds of location, respectively, in which four $\mathrm{Ca}^{2+}$ occupy the $\mathrm{Ca}$ ( I ) positions at $\mathrm{z}=0$ and $\mathrm{z}=1 / 2$, respectively, the locations of $6 \mathrm{Ca}^{2+}$ are in the $\mathrm{Ca}$ (II) positions at $\mathrm{z}=1 / 4$, and $\mathrm{z}=$ $3 / 4$, respectively, the three ligand center is composed of three $0_{3}$ and $6 \mathrm{PO}_{4}^{3-}$ ligands are located at $\mathrm{z}=1 / 4$, and $\mathrm{z}$ $=3 / 4$, which are located on a plane. The tetrahedral structure of $\mathrm{PO}_{4}^{3-}$ makes the HAP have a good stability. Its structure is shown in Figure 1. 


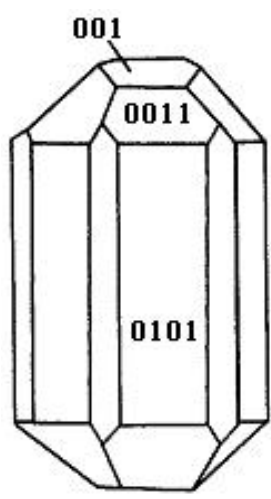

Figure 1. The space structure of hydroxyapatite

There are many methods in the preparation of nanohydroxyapatites powder, which can be divided into wet and dry methods. The wet method includes precipitation method, hydrothermal method, sol-gel method, ultrasonic synthesis, micro-emulsion method and ultrasonic synthesis method, and so on. The soldi-state reaction method belongs to the dry method. Sol-gel method is a new method of preparation of nano-hydroxyapatites in recent years. In this paper, we use the sol-gel method to prepare the nano-hydroxyapatites material, their physical and biological properties as well as their mechanism of absorption with amino acids are inspected by UV spectrophotometry, X-ray diffraction (XRD) phase analysis, infrared spectrum of absorption analysis, fluorescence spectrum and MTT method.

\section{Experimentall Methods and Results}

\subsection{Experimental Method}

The sol-gel method is used to synthesize high purity nano-hydroxyapatites ceramic powder, in which chemical reagents of diammonium hydrogen phosphate, calcium nitrate and urea are used. In this process of synthesis $6.6 \mathrm{~g}$ (0.05 mol) diammonium hydrogen phosphate are prepared into a $150 \mathrm{ml}$ solution, in which $19.72 \mathrm{~g}(0.08352$ mol) calcium nitrates are added according to the molar ratio of $\mathrm{Ca} / \mathrm{P}=1.67$. The complex solution is acted by the magnetic stirrer. After aging for 3 hours, the urea is added into the complex solution to remove $\mathrm{NO}^{3-}$, thus the sol state of the complex solution is formed through regulating its $\mathrm{pH}$ value to 9 in the condition of alkaline environment. After aging for two days, the sol becomes a white jelly-like gel. The gel is placed into the microwave oven to dry for about $10-20 \mathrm{~min}$, which again is repeatedly washed to remove $\mathrm{NH}_{4} \mathrm{NO}_{3}$. Residual samples are sintered at $1100^{\circ} \mathrm{C}$ for about 2 hours, thus producing pure nanohydroxyapatites powders through this process. Experimental devices used in this research consist of constant temperature bath, sinter, controlled device of sinter, semi-circular at the end of formwork table, precision $\mathrm{pH}$

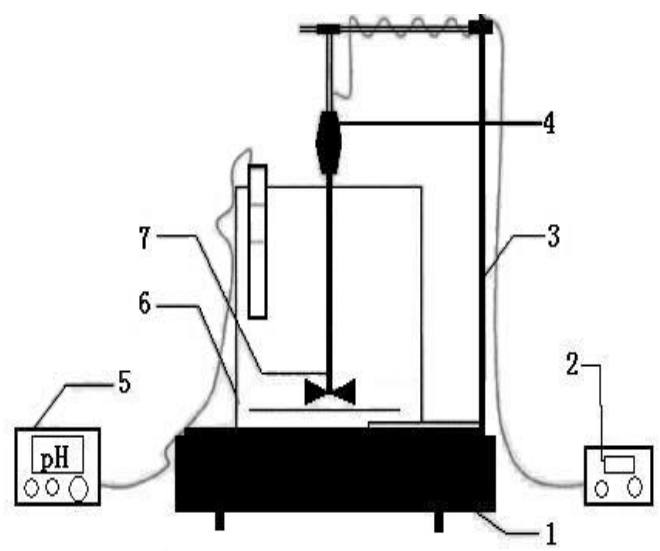

Figure 2. The experimental device of sol-gel synthesis of hydroxyapatite, where 1 is the constant temperature bath, 2 is the controlled device of sinter, 3 is semi-circular at the end of formwork table, 4 is a blender, 5 is the precision $\mathrm{pH}$ meter, 6 is the flat-bottomed beaker, 7 is the reaction solution

meter, flat-bottomed beaker, 708-type high-temperature silicon carbide oven and reaction solution composition, which is shown in Figure 2. The nano-hydroxyapatites powder prepared is placed into the different amino acid solutions, from which we extract the supernatant through centrifugation after about 40 minutes. Their composition and structure are analyzed and inspected by UV spectrophotometry, X-ray diffraction (XRD) phase analysis, Xray diffraction spectra (XDS) analysis and infrared spectrum of absorption analysis. In this experiment the aspartic acid, glutamic acid, asparagine, glutamine, potassium bromide and ethanol are also used.

\subsection{Experimental Results}

\subsubsection{The Absorbed Effect of Nanohydroxyapatites on the Amino Acid Molecules}

1) The examination of the absorbed effect by absorption of ultraviolet light.

About $50 \mathrm{mg}$ of aspartic acid, asparagine and glutamine crystalline powders are diluted to have the solutions of concentrations of about $0.25 \mathrm{mg} / \mathrm{mL}$ through adding pure water, respectively. The spectra of ultraviolet light for the three kind of amino acid solutions are measured by UV spectrometer with 190-400 $\mathrm{nm}$ wavelengths and the results are shown in Figure 3. Figure $\mathbf{3}$ shows that the solution of aspartic acids has two absorption peaks at $196 \mathrm{~nm}$ and $227.0 \mathrm{~nm}$, but the solution of asparagine has three absorption peaks at $199 \mathrm{~nm}, 203 \mathrm{~nm}$, and $268.6 \mathrm{~nm}$ and the glutamine solution has also three absorption peaks at $199 \mathrm{~nm}, 203 \mathrm{~nm}$, and $227.16 \mathrm{~nm}$.

Subsequently, $25 \mathrm{mg}$ of aspartic acid, asparagines, glutamine and glutamic acid were diluted in the pure water to obtain the standard solutions for evaluation. The solutions were shaken to have uniform solutions. From these solutions, we extract the standard solutions of $4 \mathrm{~mL}$, 


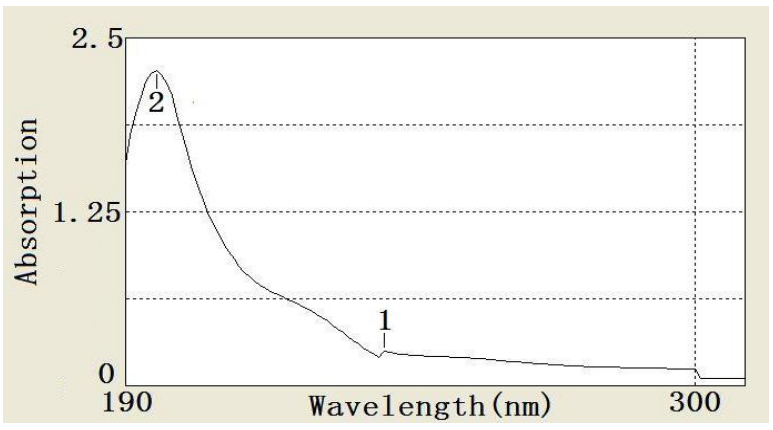

(a)

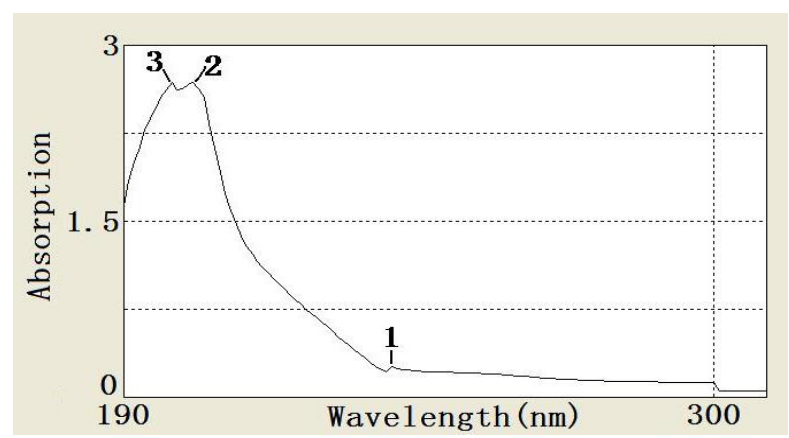

(b)

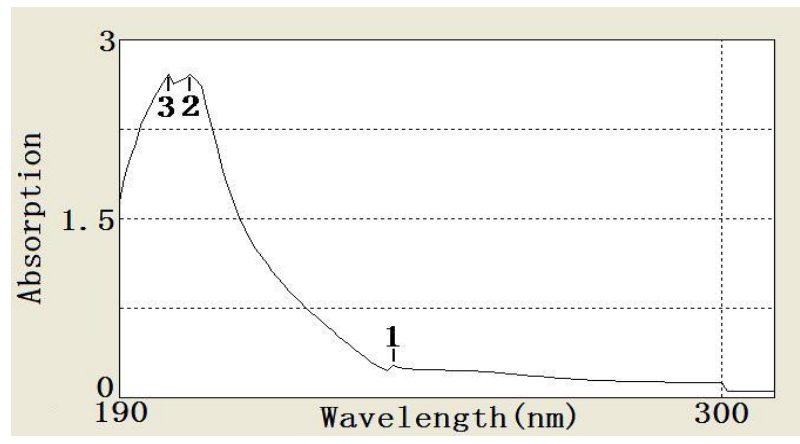

(c)

Figure 3. The ultraviolet absorption spectrum of crystalline aspartic acid (a), asparagine (b) and glutamine (c)

Table 1. The OD values of some amino acid solutions contained in deionized water

\begin{tabular}{ccccc}
\hline \multicolumn{5}{c}{ OD values } \\
\hline $\begin{array}{r}\text { Aminoac- } \\
\text { ids }(\mathrm{mg} / \mathrm{ml})\end{array}$ & $\begin{array}{c}\text { aspartic } \\
\text { acid }\end{array}$ & $\begin{array}{c}\text { gluta- } \\
\text { mine }\end{array}$ & $\begin{array}{c}\text { glutamic } \\
\text { acid }\end{array}$ & $\begin{array}{c}\text { aspar- } \\
\text { agine }\end{array}$ \\
0.05 & 0.321 & 0.205 & 0.108 & 0.200 \\
0.10 & 0.446 & 0.423 & 0.152 & 0.413 \\
0.125 & 0.492 & 0.539 & 0.175 & 0.509 \\
0.1875 & 0.615 & 0.787 & 0.261 & 0.757 \\
0.25 & 0.732 & 1.030 & 0.323 & 0.988 \\
\hline
\end{tabular}

$8 \mathrm{~mL}, 10 \mathrm{~mL}, 15 \mathrm{~mL}$ to prepare further as $20 \mathrm{~mL}$ diluted solutions with concentrations of $0.05 \mathrm{mg} / \mathrm{mL}, 0.10 \mathrm{mg} / \mathrm{mL}$, $0.125 \mathrm{mg} / \mathrm{mL}, 0.1875 \mathrm{mg} / \mathrm{mL}$, respectively, through adding deionized water. Their optical density (OD) values are measured using ultraviolet spectrometer. The results obtained are given in Table 1. The relationship between the optical density values and concentrations of amino acid molecules can be obtained from the values in Table 1 using EXCEL software. Finally it was found that they satisfy the following formulae, $\mathrm{Y}=2.0251 \mathrm{X}+02326$ for the aspartic acid, $\mathrm{Y}=4.1141 \mathrm{X}+0.0105$ for the asparagine, $\mathrm{Y}=1.1115 \mathrm{X}+0.0454$ for the glutamine, and $\mathrm{Y}=$ $3.9305 \mathrm{X}+0.0133$ for the glutamic acid, respectively, where $\mathrm{Y}$ denotes the optical density value and $\mathrm{X}$ is the concentration of the amino acid.

The properties of the amino acids absorption of nanohydroxyapatites can now be determined.

a) $25 \mathrm{mg}$ HAP was added into $8 \mathrm{~mL}$ aspartic acid solution with concentration of $0.1875 \mathrm{mg} / \mathrm{m}$, and three samples of the supernatant of the solution were extracted through centrifugation for 30 minutes and their OD values were measured as $0.530,0.529,0.536$, respectively, and an average value of 0.532 . From the above formula we can derive the concentration of aspartic acid to be $\mathrm{X}=0.1478$. Then the absorption values of the aspartic acid by the nano-hydroxyapatites powder can be found to be $\mathrm{A}=8 \times$ $(0.1875-0.1478) \times 100 / 25=1.27 \mathrm{mg} / 100 \mathrm{mg}$.

b) $25 \mathrm{mg}$ HAP was added into $8 \mathrm{~mL}$ glutamine solution with concentration of $0.1875 \mathrm{mg} / \mathrm{m}$, and three samples of the supernatant of the solution were extracted through centrifugation for 30 minutes and their OD values were measured as $0.638,0.651,0.637$, respectively, and an average value of 0.642 . From the above formula we can derive the concentration of aspartic acid to be $\mathrm{X}=0.160$. Then the absorption values of the glutamine solution

$\mathrm{A}=8 \times(0.1875-0.1478) \times 100 / 25=1.27 \mathrm{mg} / 100 \mathrm{mg}$.

c) $25 \mathrm{mg}$ HAP was added into $8 \mathrm{~mL}$ asparagine solution with concentration of $0.1875 \mathrm{mg} / \mathrm{m}$, and three samples of the supernatant of the solution were extracted through centrifugation for 30 minutes and their OD values were measured as $0.737,0.734,0.735$, respectively, an average value of 0.735 . From the above formula we can derive the concentration of aspartic acid to be $X=0.176$. The absorption values of the asparagine solution by the nanohydroxyapatites powder can be found to be $\mathrm{A}=8 \times$ $(0.1875-0.176) \times 100 / 25=0.368 \mathrm{mg} / 100 \mathrm{mg}$.

d) $25 \mathrm{mg}$ HAP was added into $8 \mathrm{~mL}$ glutamic acid solution with concentration of $0.1875 \mathrm{mg} / \mathrm{m}$, and three samples of the supernatant of the solution were extracted through centrifugation for 30 minutes and their OD values were measured as $0.206,0.204,0.210$, respectively, an average value of 0.207 . From the above formula we can derive the concentration of aspartic acid to be $\mathrm{X}=0.145$. Then the absorption values of the glutamic acid solution by the nano-hydroxyapatites powder can be found to be A 
$=8 \times(0.1875-0.145) \times 100 / 25=1.36 \mathrm{mg} / 100 \mathrm{mg}$.

The above results indicate clearly that the nano-hydroxyapatites powders can absorb the amino acid molecules, although the absorbed amounts are not the same for different amino acid molecules. We know from this result that the capability of the nano-hydroxyapatites absorbing the glutamic acids is most strong, next is aspartic acid and glutamine. Why is this? As it is known, the four amino acid molecules are all acidic and have certain charges, thus they can interact with other charged matter, but the acidity of the glutamic acid is most strong relative to others because it contains again the gamma-carboxyl, which can interact with calcium ions and other charged ions. On the other hand, we know that the surface of the nano-hydroxyapatites has some calcium ions and phosphates, which can interact with and absorb acidic amino acid molecules, specially the glutamic acids. Therefore, it is not difficult to conclude that the stronger of acidity of amino acid molecules, the better is the absorption capability of the nano-hydroxyapatites.

2) The X-ray diffraction (XRD) phase analysis for the effect of amino acids absorption of the nano-hydroxyapatites. $200 \mathrm{mg}$ nano-hydroxyapatites were added into the saturated solution consisting of $100 \mathrm{~mL}$ glutamic acid and $100 \mathrm{~mL}$ deionized water to obtain the nano-hydroxyapatites complex solution, from which we extract the insoluble matter through filtering at room temperature after two days. The pure experimental samples of the insoluble matter are obtained through repeated washing to remove the excessive amount of glutamic acid on the surface of nano-hydroxyapatites by using the deionized water, and dried for about 1 hour. The X-ray diffraction pattern of the nano-hydroxyapatites complex with and without glutamic acids obtained is shown in Figure 4, respectively. Figure 4 shows that some peaks of the X-ray diffraction of the nano-hydroxyapatites complex with glutamic acids become higher and sharper, but another peak has disappeared relative to the results obtained from the samples without glutamic acids. This indicates that the crystallization degree of nano-hydroxyapatites with glutamic acids becomes much higher.

3) Infrared spectrum of absorption from the complex of nano-hydroxyapatites and amino acid molecules.

In this experiment the solutions consisting of $200 \mathrm{mg}$ nano-hydroxyapatites and $100 \mathrm{~mL}$ saturated solution of glutamic acid are prepared; their infrared spectra of absorption are collected by NexusFT-IR670 spectrometer. Figure 5 shows the infrared spectra of absorption of the solutions in the range of $40 \sim 4000 \mathrm{~cm}^{-1}$ at different times at $0 \mathrm{~min}, 0.5 \mathrm{~min}, 2 \mathrm{~min}, 5 \mathrm{~min}, 10 \mathrm{~min}$, respectively. It was found that Figure 5 is different from Figure 2. Figure 5(b) shows this difference, in which a new peak of $1028 \mathrm{~cm}^{-1}$ occurs in the complex solution, which indicates that the glutamic acid have been incorporated with the nano-hydroxyapatites. Since the peak is increased with increasing time of reaction, which can be seen from Figure 5(b), thus we can show that the incorporation capability and the amount the glutamic acids into the nano-hydroxyapatites are increased with increasing time of reaction. Obviously, the incorporation is due to the interaction of the $\mathrm{PO}_{4}^{3-}$ group or $\mathrm{Ca}^{+2}$ group with glutamic acids with certain dipole moment. With increasing time, more and more glutamic acid molecules are shifted into nano-hydroxyapatites structure, which result in the increases in the strength of infrared absorption as shown in Figure 5(b).

4) The changes of state of amino acid molecules due to the absorption of nanohydroxyapatites.

From the above study we know that the nanohydroxyapatites can absorbed the amino acid molecules. Then the structure and conformation of amino acid molecules will be changed, when they are absorbed. We here study the changes of state of glutamic acid using re-crystalline method. In this experiment, we simultaneously added the minor nano-hydroxyapatites (nanoHAP) powder into a water solution of crystalline amino acid molecules to form a hybrid solution, which is called an experimental group, but a controlled group is only composed of the water and crystalline amino acid molecules without the nanohydroxyapatites. Subsequently, the hy-

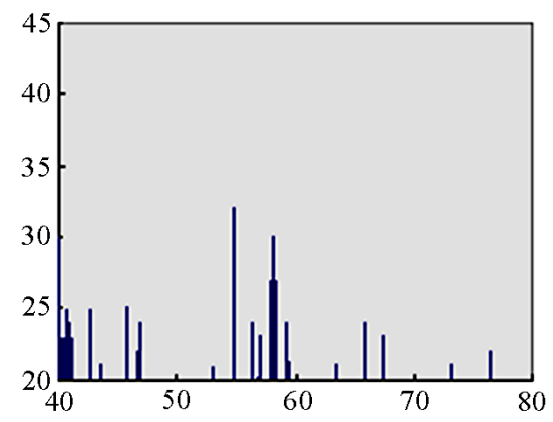

(a)

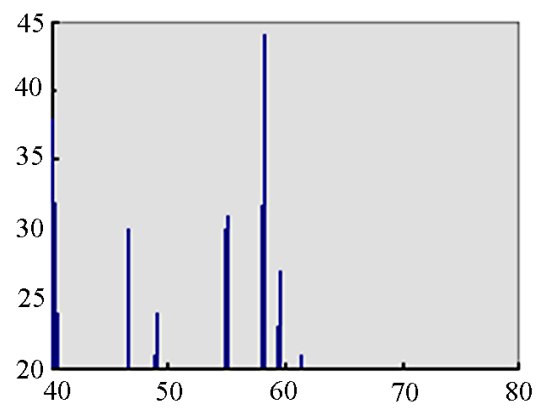

(b)

Figure 4. X-ray diffraction pattern of the nano-hydroxyapatites complex without glutamic acid (a) and with glutamic acid (b) 


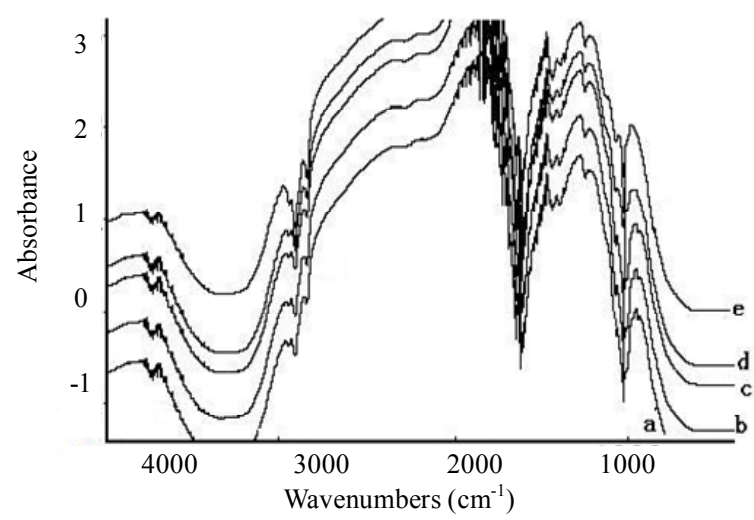

(a)

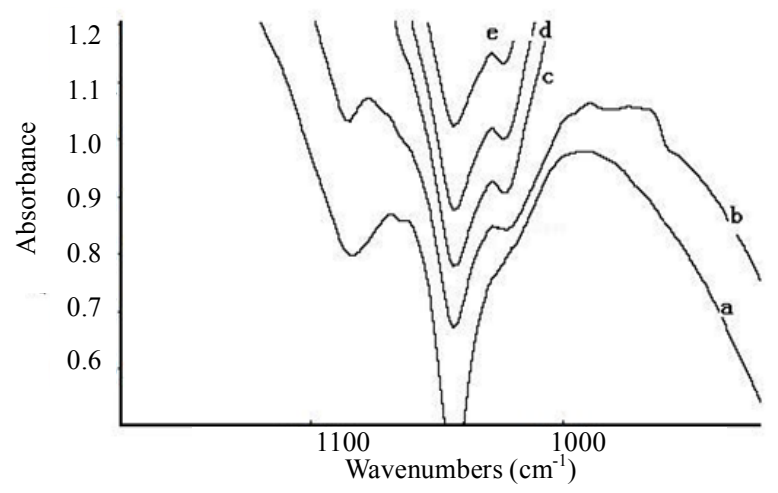

(b)

Figure 5. Infrared spectra of absorption of the solutions consisting of nano-hydroxyapatites and glutamic acid molecules at different times, where $a$ is the result of $0 \mathrm{~min},(\mathrm{~b})$ is the $0.5 \mathrm{~min}, \mathrm{c}$ is the $2 \mathrm{~min}, \mathrm{~d}$ is the $5 \mathrm{~min}$, $\mathrm{e}$ is the $10 \mathrm{~min}$. Fiure 5(b) expressed the change of infrared spectra in the range of $1000-1100 \mathrm{~cm}^{-1}$.

brid solutions in the experimental and controlled groups crystallize simultaneously under the same conditions by decreasing the temperature. Finally, the crystals of the amino acid molecules and amino acid molecules with the nanohydroxyapatites were observed by optic microscope to determine their crystalline shapes. The results are shown in Figure 6. From this figure, it can be seen clearly that the re-crystalline state and shape of the glutamic acid were changed after interaction with the nanoHAP, when compared with that of the controlled group. In the experimental group, it is evident that the head parts of the re-crystalline glutamates become a cone shape from a cylinder shape. This may be due to the fact that these HAPs become crystalline nucleuses when the glutamates are re-crystallized. Thus, the re-crystalline states in the experimental and controlled groups are different because the shape and feature of the nanoHAPs differ from that of the glutamaic acid molecules. This verifies again that the nanoHAP can interact with amino acid molecules as mentioned above.

\subsubsection{The Influence of Nano-Hydroxyapatites on the Proliferation of Cells and its Toxicity}

The MTT colorimetric method was often used to study the influences of the nanomaterials on the activity and growthof cells, so that their toxicity can be determined from such a study [7]. In the MTT colorimetric method, the coloration substance used is MTT [7], which is 3-(4, 5-dimethylthiazol 2-yl)-2, 5 diphenyltetrazolium bromide and a type of dye that can accept the hydrogen atoms. When the externally applied yellow tetrazolium salt 3-(4, 5-dimethylthiazol 2-yl)-2, 5 diphenyltetrazolium bromide (MTT, American) is taken up by the mitochondria of a cell, it will be reduced to a blue insoluble crystalline matter through a reaction with an amber acid dehydrogenase in the cell. The insoluble matter will be deposited in the live cells, but not in the the dead cells. However, these depositions can also be dissolved by injecting dimethylsulfoxide (DMSO). The amount of dissolution is proportional to the number of cells which participated in the reaction. Thus the number of cells participating in the process can be obtained indirectly by measuring the strength of absorption of the light with at a wavelength of $490 \mathrm{~nm}$ using an enzymatic immunoassay instrument and spectrophotometer (American). Osteoblast cells (MG63) were chosen to study the toxic effects of the nano-hydroxyapatites on the proliferation of the cells in our experiment. The advantages of this method are that it is

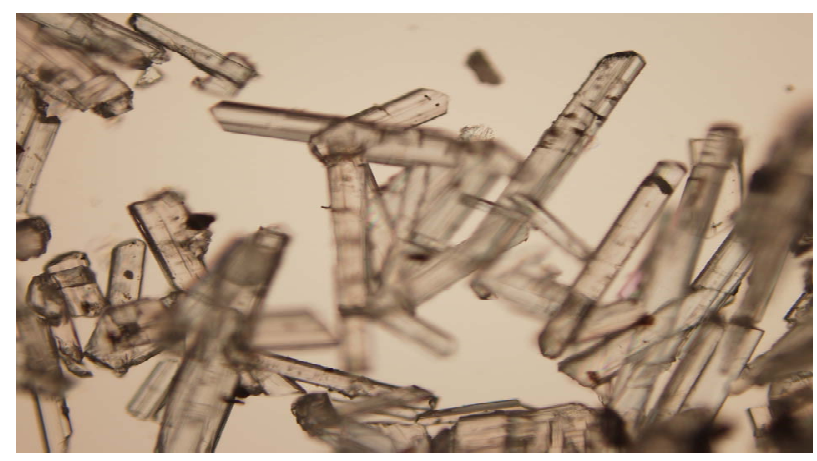

(a)

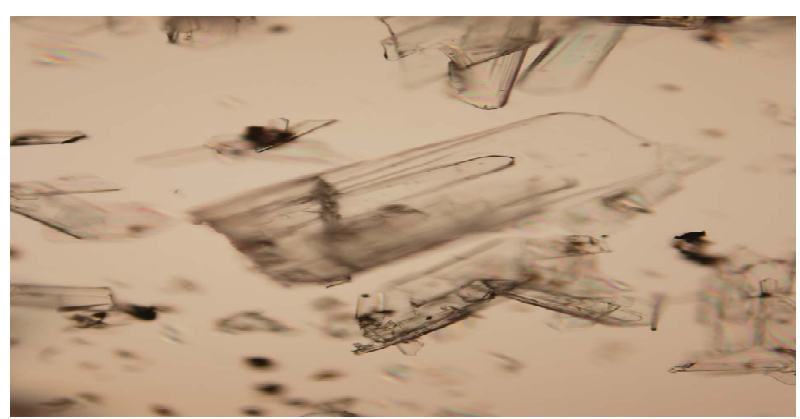

(b)

Figure 6. (a) crystalline state of glutamate (b) crystalline state of glutamate + nanoHAP 
convenient, highly accurate, and has a high sensitivity of measurement and very good reproducibility. The experimental process and method are described as follows.

1) The culture of cells. The MG63 cells were grown in a $5 \% \mathrm{CO}_{2}$ enriched incubator with a temperature of $25^{\circ} \mathrm{C}$ in RPMI1640 media (Hyclone, American) supplemented with 5\% fetal calf serum (FCS, Biological Industries, BaiAn, China) [7]. Using microscopic inspection, it was verified that there were no contaminating cells at the third passage of cell culture.

2) The experimental process. In all experiments, $4 \times$ $10^{5}$ cells per well were seeded into 60 -well micro-plates and allowed to grow continually under the conditions described above. The $100 \mu \mathrm{L} /$ well foster liquids containing the fetal calf serum and $1 \mathrm{~mL} / 250 \mathrm{~mL}$ insulin liquid were added into each well, which are again separated into experimental and controlled groups. A total of $2 \mathrm{mg}$ of the nano-hydroxyapatites was added into the experimental group in a volume of $1 \mathrm{~mL}$. Subsequently, the experimental and controlled groups were placed simultaneously into the $\mathrm{CO}_{2}$ enriched incubator at $37^{\circ} \mathrm{C}$ for 24 hours. By comparing the extent of proliferation of the cells between the experimental and controlled groups, the influences of the nano-hydroxyapatites on the states of proliferation of the MG63 cells can be determined.

3) Measurement of the extent of proliferation of the MG63 cells. The extent of the proliferation of the MG63 cells was determined using the MTT colorimetric method. First, the changes in the mitochondrial dehydrogenase activity with increasing number of cells were measured. A volume of $100 \mu \mathrm{L} /$ well of MTT solution was prepared in PBS (5 mg/mL) and further diluted (10\%) in RPMI 1640. The cell growth medium was aspirated and $100 \mu \mathrm{L}$ of the MTT solution was added into each well. The MG63 cells were further incubated for $4 \mathrm{~h}$ at $37^{\circ} \mathrm{C}$. The excess MTT solution was removed and $100 \mathrm{~L} /$ well DMSO was added to dissolve the blue crystalline matter that had been formed in the cells.

4) The experimental results. The density (OD) of the DMSO solutions in each well in the controlled and experimental groups was measured spectro-photometrically at $490 \mathrm{~nm}$ by a DG3022 enzymatic-immunoassay instrument using the MTT method. The OD values of the experimental and controlled groups are shown in Table 2. Thus, the cell proliferation rate (CPR) of the MG63 cells after treatment with the nano-hydroxyapatites can be determined according to the formula

$$
\mathrm{CPR}=\left[\left(\mathrm{D}_{\exp }-\mathrm{D}_{\text {con }}\right) / \mathrm{D}_{\text {con }}\right] \times 100 \% \text {, }
$$

where $D_{\exp }$ is the value of optical density of the experi mental group, and $\mathrm{D}_{\text {con }}$ is the value of optical density of the control group. The values of relative cell proliferation tained using:

$$
\mathrm{RCPR}=\left(\mathrm{D}_{\text {exp }} / \mathrm{D}_{\text {con }}\right) \times 100 \%
$$

It is evident that there are differences in the parameters between the experimental and controlled groups $(\mathrm{P}<0.05)$, but the degree of toxicity of the nano-hydroxyapatites to the MG63 cells is minor.

Table 2 shows that the nano-hydroxyapatites and the complex of nano-hydroxyapatites + nanoCrO 2 (HAP+ $\mathrm{CrO}_{2}$ ) can all restrain the proliferation of cells, but their toxicities are all first degree or minor; but the relative cell proliferation rate of MG63 cells decreases with increasing time of growth under the influence of the nano-hydroxyapatites and complex nano-hydroxyapatites + nano$\mathrm{CrO}_{2}$ (nano(HAP+ $\left.\mathrm{CrO}_{2}\right)$ ). Meanwhile, we find that the influence of complex nano $\left(\mathrm{HAP}+\mathrm{CrO}_{2}\right)$ on the proliferation of growth of cells are minor compared to that with nano-hydroxyapatites. This is a new and interesting result.

\subsection{The Properties of Interaction of Nano-Hydroxyapatite with the Proteins of Human Serum Albumin}

We prepare the complex solutions of human serum albumin(HSA) and nano-hydroxyapatite, the latter is added into the solution composed of HAS and physiological saline with the concentration of $2.5 \%$, We prepare the pure HBA and three samples with different Mol ratios of concentration of nano-hydroxyapatite and HBA, which are $1 / 3,1 / 13$ and $1 / 130$ in the complex solution, respectively. We used 7000-fluorescent spectrometer made by Japan and Nexus FT-IR670 infrared spectrometer to measure and collect the fluorescent and infrared spectra of the supernatants which are extracted from the solutions of three samples through the filter after soaking of 10 days. The fluorescent spectra of the four samples are shown in Figures $\mathbf{7}$ and $\mathbf{8}$. Figure 7 represents clearly that there are all a main peak at $348.8 \mathrm{~nm}$ in the four samples, their strengths increase and half width of peak decrease with increasing quantity of nano-hydroxyapatites added in the complex solutions of HBA, the changes of normalized half width of the peak at $348.8 \mathrm{~nm}$ for the three complex solutions shown in Figure 8. For the complex of concentration of $1 / 3$, its half peak width is $110.4 \mathrm{~nm}$, it is 112.8 $\mathrm{nm}$ for the complex of $1 / 13$, but it is $114.2 \mathrm{~nm}$ for complex of $1 / 130$.

This means that the nano-hydroxyapatites are absorbedby HBA and vary greatly the structure of the latter. Meanwhile, we found that a new peak at $548.8 \mathrm{~nm}$ occurs in the fluorescent spectra of four samples, but the strengths of the three complex solutions containing the nano-hydroxyapatites are larger than that of pure HBA, which are shown in small figure inserting Figure 7. This shows that the structure of HBA is changed, some new activity centers occur in it due to the absorption and addition of the nano-hydroxyapatites.

Figures 9-13 shows the infrared spectra of absorption of nano-hydroxyapatite, HBA and their complex solutions with Mole ratios of concentration of $1 / 3,1 / 13$ and $1 / 130$, respectively. Figure 9 shows the infrared spectrum of 


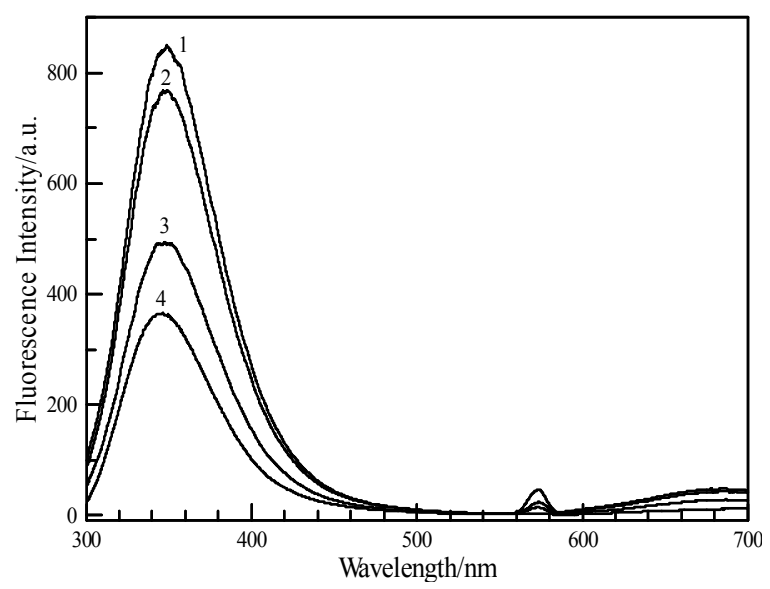

(a)

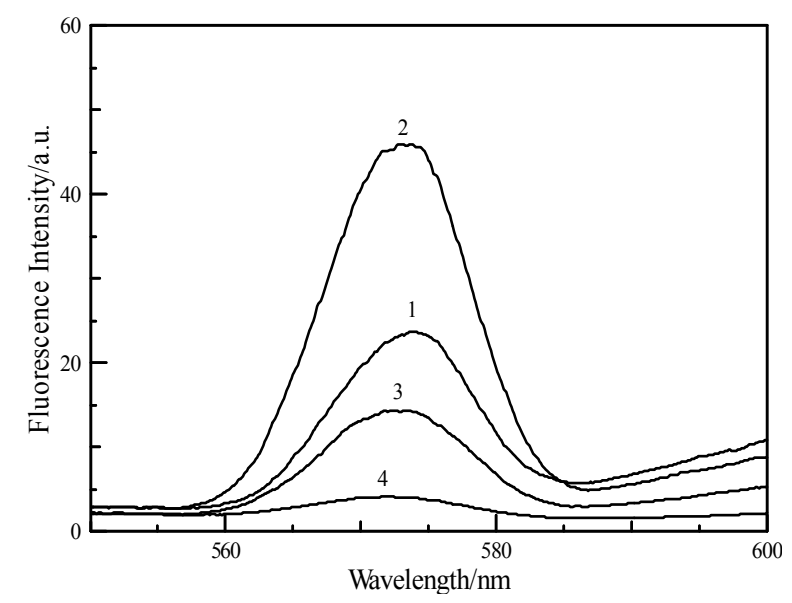

(b)

Figure 7. The fluorescence spectra of complex solutions of nano hydroxyapatite and HBA, where 1, 2 and 3 in Figure 7 (a) denote the results of the complexes with Mole ratios of concentration of $1 / 130,1 / 13$ and $1 / 3$, for hydroxyapatite in HBA, respectively, 4 is the values of pure HBA solution, Figure 7(b) shows corresponding strength of the peaks at $548.8 \mathrm{~nm}$ for the four samples

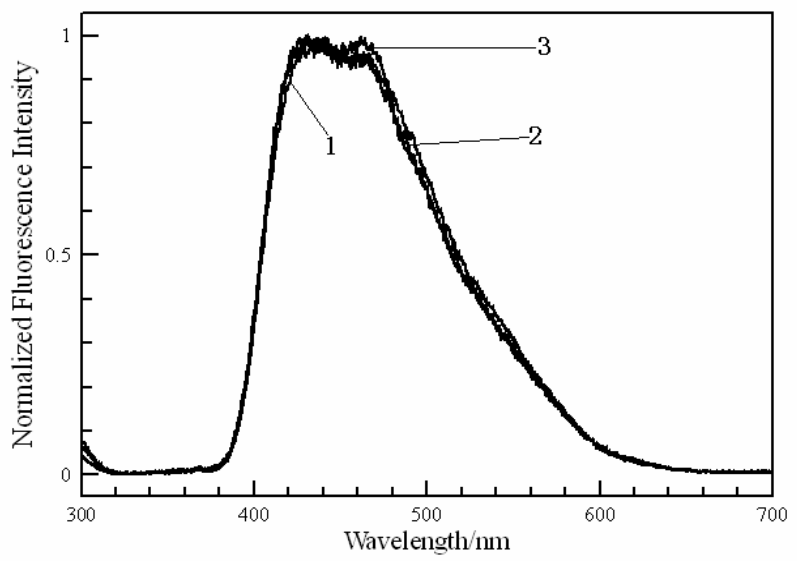

Figure 8. The changes of normalized half width of the peak at $348.8 \mathrm{~nm}$ in the fluorescent spectra of three complex solutions, where 1, 2 and 3 denote the results of complex solutions with Mole ratios of concentration of 1/3, 1/13 and 1/130 for the nano-hydroxyapatite and HBA, respectively

absorption of the nano-hydroxyapatites in the range of $400-4000 \mathrm{~cm}^{-1}$. We see from this figure there are the peaks at $1038.27 \mathrm{~cm}^{-1}$ and $964.32 \mathrm{~cm}^{-1}$, which are the characteristic peaks of $\mathrm{PO}_{4}^{3-}$, peaks at $3568 \mathrm{~cm}^{-1}$, which indicate the infrared absorption spectrum of $\mathrm{OH}$-group. and a peak at $1092.78 \mathrm{~cm}^{-1}$, which is the infrared absorption spectrum of $\mathrm{HOH}$ group. However it contains a small amount of $\mathrm{Ca}\left(\mathrm{NO}_{3}\right)_{2}$. When Figsures 11-13 compare with Figures 9 and $\mathbf{1 0}$ we found that the infrared spectra, including the number, position and strength, of the complex solutions with Mole ratios of concentration of 1/3, 1/13 and $1 / 130$ for the nano-hydroxyapatite and HBA differ those of both HBA and nano-hydroxyapatite.

This indicates that HBA absorbed the nano-hydroxyapatite, thus its structure of molecule is changed also due to the action of the nano-hydroxyapatite. This conclusion is the same with the above's.

Table 2. The optical density and relative generation rate of MG63 cells under influences of nanohydroxyapatites (HAP) and complex of nano-hydroxyapatites + nanoCrO $\mathrm{O}_{2}\left(\mathrm{HAP}+\mathrm{CrO}_{2}\right)$ with the same concentration of $5 \mathrm{mg} / \mathrm{mL}$ at different days

\begin{tabular}{|c|c|c|c|c|c|c|}
\hline \multirow[t]{2}{*}{ time } & \multicolumn{2}{|c|}{ HAP } & \multicolumn{2}{|c|}{$\mathrm{HAP}+\mathrm{CrO}_{2}$} & \multicolumn{2}{|c|}{$\begin{array}{l}\text { controlled } \\
\text { group }\end{array}$} \\
\hline & $\mathrm{P}(\%)$ & OD & $\mathrm{P}(\%)$ & OD & $\mathrm{P}(\%)$ & OD \\
\hline $1 d$ & 98.6 & $0.246 \pm 0.0596$ & 99.4 & $0.260 \pm 0.0505$ & 100 & $0.275 \pm 0.0513$ \\
\hline $3 d$ & 94.9 & $0.290 \pm 0.0519$ & 97.5 & $0.435 \pm 0.0415$ & 100 & $0.500 \pm 0.540$ \\
\hline $5 d$ & 93.7 & $0.299 \pm 0.0236$ & 95.9 & $0.539 \pm 0.2732$ & 100 & $0.619 \pm 0.258$ \\
\hline $7 \mathrm{~d}$ & 92.6 & $0.312 \pm 0.0230$ & 93.6 & $0.661 \pm 0.0715$ & 100 & $1.002 \pm 0.460$ \\
\hline
\end{tabular}




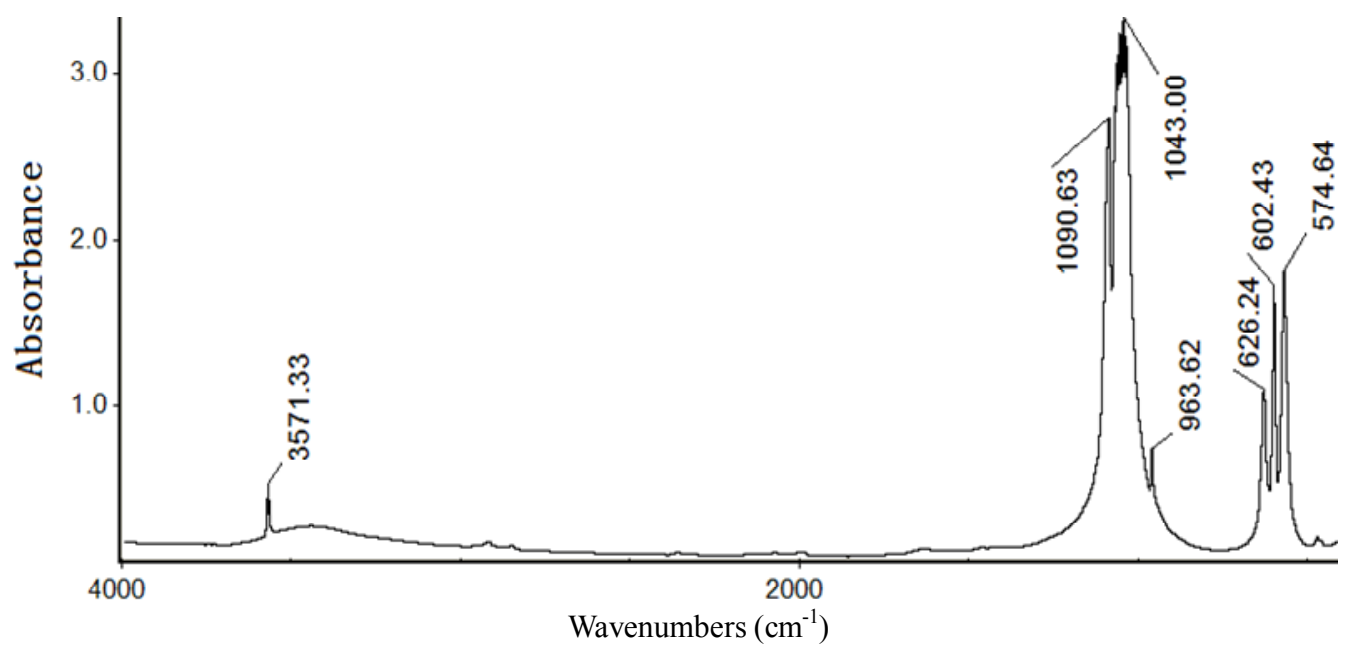

Figure 9. The infrared spectrum of absorption of the nano- hydroxyapatite

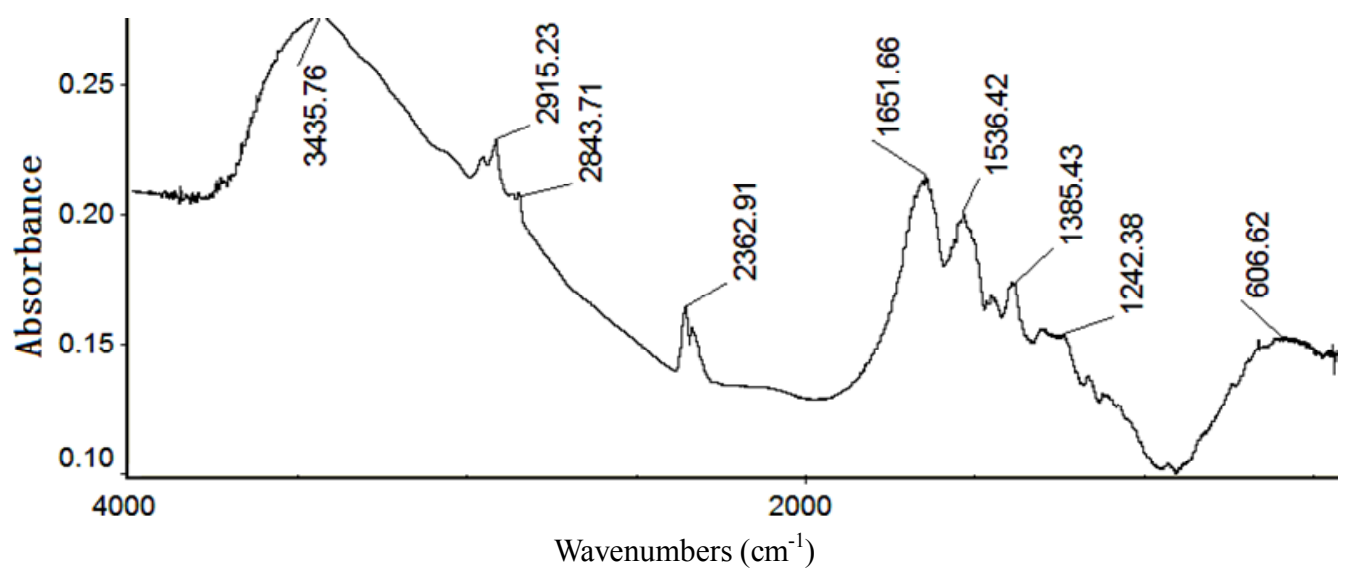

Figure 10. The infrared spectrum of HBA

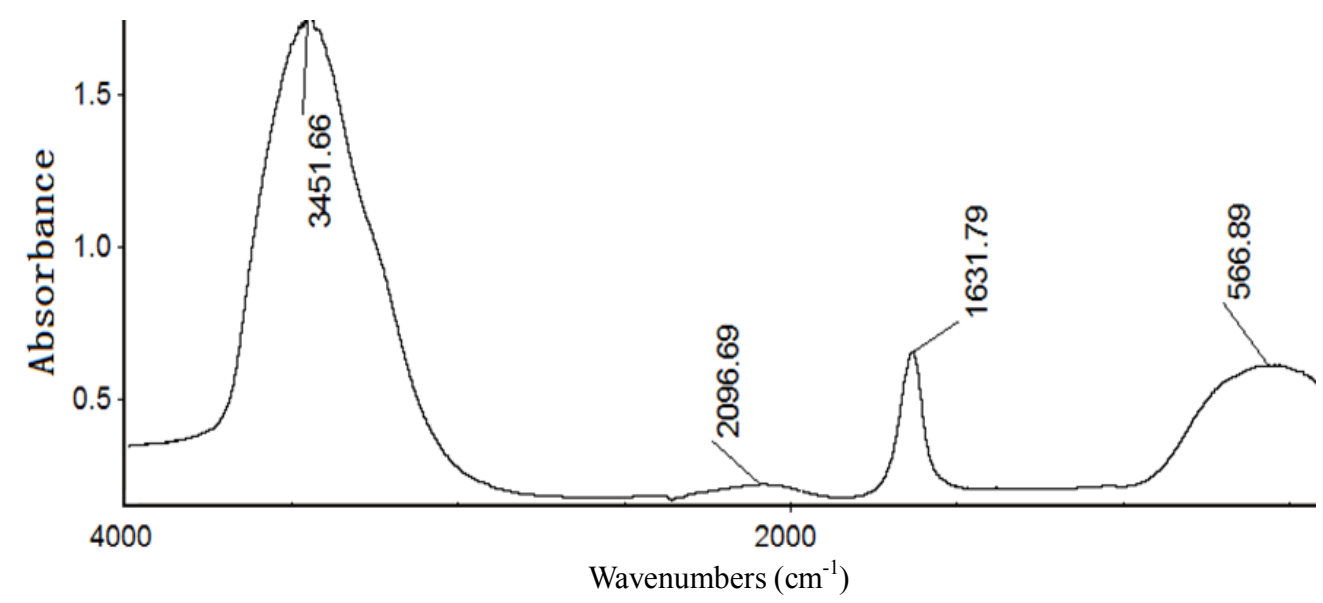

Figure 11. The infrared spectrum of complex solution with Mole ratios of concentration of $1 / 3$ for the nano-hydroxyapatite and HBA. 


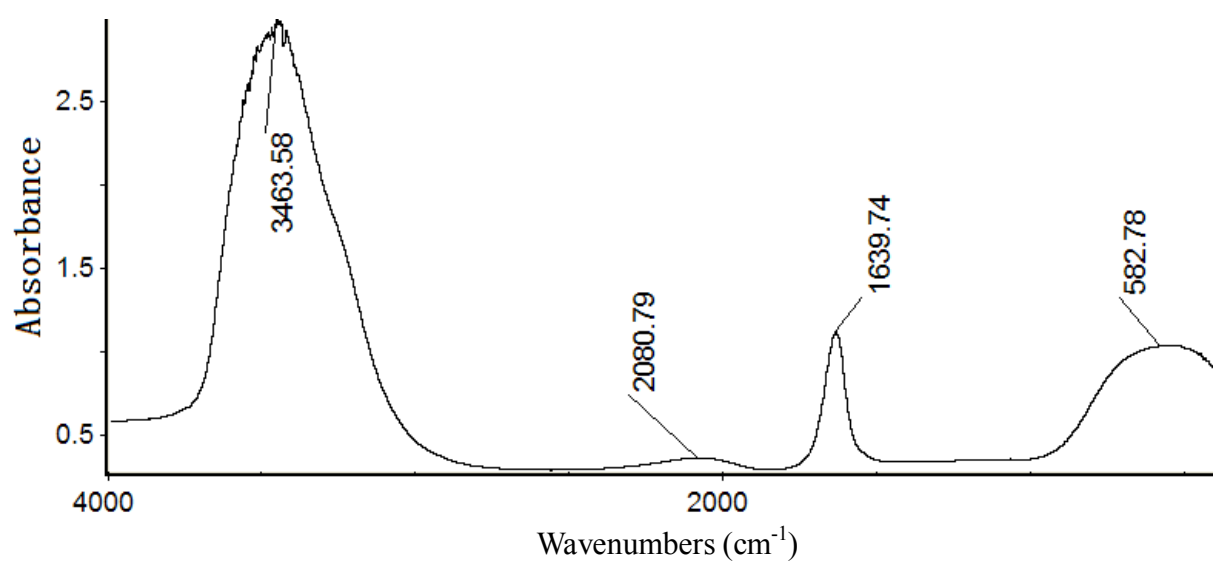

Figure 12. The infrared spectrum of complex solution with Mole ratios of concentration of 1/13 for the nano-hydroxyapatite and HBA

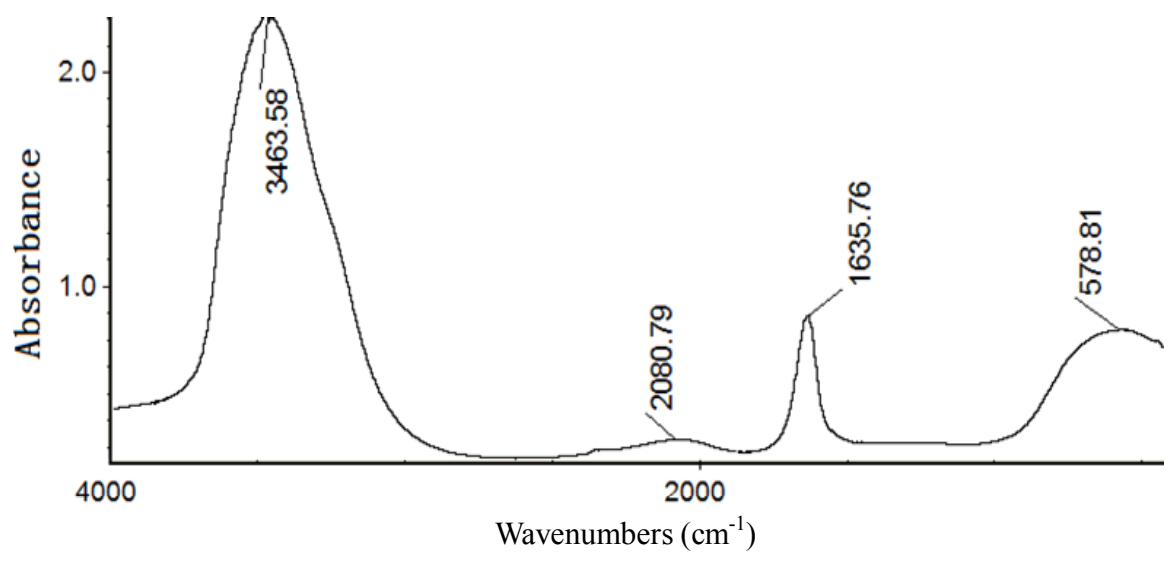

Figure 13. The infrared spectrum of complex solution with with Mole ratios of concentration of 1/130 for the nano-hydroxyapatite and HBA

\section{Conclusions}

The paper investigate the biological effects of nanohy-droxyapatites using ultraviolet absorption spectrum, X-ray diffraction (XRD)structure analysis, infrared spectrum of absorption and MTT method. The nanohydroxyapatites we used in this experiment are prepared an made by using Sol-gel method, in which the parameters of process and reaction are controlled as: $\mathrm{PH}>9, \mathrm{Ca} / \mathrm{P}=$ 1.67 , sintering temperature of $1100^{\circ} \mathrm{C}$ and sintering time 2 hours. From this investigation the following conclusions can be obtained:

1) The nanohydroxyapatites can interact and adsorb the amino acid molecules, but the absorb quantities are not same for different amino acid molecules. It was found that the stronger of acidity of amino acid molecules, the better is the absorption capability.

2) The nanohydroxyapatites and complex of nano$\mathrm{HAP}+$ nanoCrO $\mathrm{Cr}_{2}$ can all restrain the proliferation of cells, but their toxiciteis are all first degree or minor. Meanwhile the restrained effect of the latter is smaller than that of the former, although they can decrease the relative proliferation rate of cells. This shows clearly that the incorporation of the nanohydroxyapatites with nanoCrO $\mathrm{Cr}_{2}$ changes the biological activity of the former. This is a new and interesting result.

3) The nanohydroxyapatites can absorb the human serum albumin and can also change its molecular structure.

\section{Acknowledgments}

The authors would like to acknowledge the Major State Basic Research Development Program (973 program) of China for their financial support (Grant No. 2007CB9 36103).

\section{REFERENCES}

[1] R. R. Liu, X. Mao, Q. C. Yu, et al., "Preparation of Bioactive Nanohydroxya-Patite Coating for Artificial Cornea," Current Applied Physics, Vol. 7, No. 1, 2007, pp. 
85-89.

[2] Z. J. Wu, L. P. He and Z. Z. Chen, "Fabrication and Characterization of Hydroxyapa-Tite/A12O3 Biocomposite Coating to Titanium," The Transactions of Nonferrous Metals Society of China, Vol. 16, No. 3, 2006, pp. 259266.

[3] C. S. Chai and B. Bennissan, "Bioactive Nanocrystalline Sol-Gel Hydroxyapatite Coatings," Journal of Materials Science: Materials in Medicine, Vol. 10, No. 4, 1999, pp. 465-469.

[4] F. L. Yu, Y. Q. Zhou, W. K. Zhang and J. Y. Ma, "Preparation and Development Prospects of Research of Hy- droxyapatite Biomaterials," Ceramics, Vol. 23, No. 2, 2006, pp. 6-11.

[5] A. Bennick, M. Cannon and G. Madapallimattam, "He Nature of the Hydroxyapatite-Binding Site in Salivary Acidic Proline-Rich Proteins," Biochemical Journal, Vol. 183, No. 1, 1979, pp. 115-126.

[6] Q. Ye, R. Hu, Z. Y. Lin and C. J. Lin, "Hydroxyl Apatite and Bovine Serum Albumin Interactions in Situ Infrared Spectroscopy," Chemical Journal, Vol. 26, No. 8, 2006, pp. 946-950.

[7] Z. Q. Situ and J. Z. Wu, "Cluture of Cell," World Book Publishing Company, Beijing, 1996, pp. 186-187. 